\title{
PATTERNS OF THERMOMAGNETIC CONVECTION CAUSED BY LONG- OR SHORT-WAVE MODULATION OF THE MAGNETIC FIELD
}

\author{
A. Lange, S. Odenbach \\ TU Dresden, Institute of Fluid Mechanics, Chair of Magnetofluiddynamics, \\ 01062 Dresden, Germany
}

\begin{abstract}
An analysis of the thermomagnetic convection in a horizontal layer of magnetic fluid subjected to a spatially and symmetrical modulated magnetic field is presented. Both the magnetic field and the temperature gradient are oriented vertically. The base state is a convective one formed by a double vortex, which reflects the symmetrical modulation and appears for any nonzero magnetic field. Depending on the type of modulation, different shapes of convection rolls appear: either horizontally stretched convection rolls for long-wave modulations or circular shaped ones for short-wave modulations.
\end{abstract}

Introduction. Fluidic systems, which are driven out of equilibrium by the temperature dependence of the density of the fluid, belong to the set of classical pattern forming systems $[1,2]$. By using a magnetic fluid (MF) as a working substance, new ways open up to generate patterns. Magnetic fluids, which are stable colloidal suspensions of ferro- or ferrimagnetic nanoparticles dispersed in a carrier liquid, are superparamagnetic fluids. Their magnetization generates a magnetic force in interaction with an applied magnetic field, which can drive the system out of equilibrium, too. Thus, in a horizontal layer of MF subjected to a vertical gradient of temperature and a vertical magnetic field convection can be triggered in two different ways. A detailed description of the two processes can be found in [3].

The study of thermomagnetic convection was started with the case of static external magnetic fields [4-6]. The focus was directed to the interaction of the thermomagnetic convection with the density driven one [4], where the resulting flow patterns of aligned as well as hexagonal and irregular oriented convection rolls were visualized in $[5,6]$. Widening the focus of interest, the modulations of the magnetic field were first made with respect to time and lead to a parametrically driven convection [7]. A drawback of this type of excitation is that frequencies of the magnetic field above $5 \mathrm{~Hz}$ are difficult to implement limiting the prospects of variable stimulation. Therefore, the idea of a spatially modulated field appears, where such restrictions do not appear. A spatial modulation is easy to accomplish by placing sinus-like shaped iron bars in a constant magnetic field. The generic case of a symmetrical arrangement is chosen here, see Fig. 1, and studied in the rest of the paper.

1. Governing equations. A laterally infinite horizontal layer of an incompressible layer of a magnetic fluid (density $\rho$, dynamic viscosity $\eta$ ) of thickness $d$ in the $(x, y)$-plane is bounded by two boundaries in the planes $z= \pm d / 2$. The setup is heated from below with a temperature $T_{\mathrm{b}}$ for the bottom boundary, which results in a temperature difference $\delta T=T_{\mathrm{b}}-T_{\mathrm{t}}$ with respect to the top boundary with the temperature $T_{\mathrm{t}}<T_{\mathrm{b}}$. The layer is placed between two sinus-liked shaped iron bars, see Fig. 1, and the entire system is subjected to a constant external 


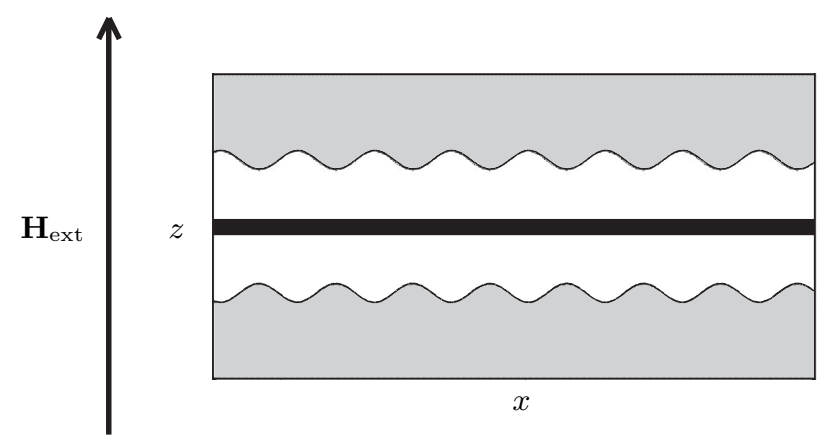

Fig. 1. Symmetric arrangement of two sinus-like shaped iron bars (gray) beneath and above a centered layer of a magnetic fluid (black) subjected to a vertical external magnetic field $\mathbf{H}_{\text {ext }}$. The white area indicates air.

magnetic field $\mathbf{H}_{\text {ext }}=\left(0,0, H_{\text {ext }}\right)$. Such a setup generates a nearly perfect vertical magnetic field, which is modulated with the wavelength $\lambda$ with respect to the horizontal coordinate $x$. With the assumption that the magnetization $\mathbf{M}$ of the magnetic fluid depends linearly on the applied magnetic field $\mathbf{H}, \mathbf{M}=\left(\mu_{r}-1\right) \mathbf{H}$, the magnetic induction is given by $\mathbf{B}=\mu_{0}(\mathbf{M}+\mathbf{H})=\mu_{0} \mu_{r} \mathbf{H}$. The relative permeability of the MF is denoted by $\mu_{r}$, and $\mu_{0}$ is the permeability of free space. The system is governed by the equation of continuity

$$
\operatorname{div} \mathbf{v}=0,
$$

the Navier-Stokes equation in the Boussinesq approximation

$$
\rho_{0}\left[\frac{\partial \mathbf{v}}{\partial t}+(\mathbf{v g r a d}) \mathbf{v}\right]=-\operatorname{grad} p+\rho \mathbf{g}+\mu_{0}(\mathbf{M} \operatorname{grad}) \mathbf{H}+\eta \Delta \mathbf{v},
$$

and the equation of heat conduction

$$
\frac{\partial T}{\partial t}+(\mathbf{v g r a d}) T=\kappa \Delta T .
$$

The density at the mean temperature $T_{0}=\left(T_{\mathrm{b}}+T_{\mathrm{t}}\right) / 2$ is denoted by $\rho_{0}$, the acceleration due to gravity by $\mathbf{g}=(0,0,-g)$, the thermal diffusivity by $\kappa$, and the linear dependence of the density on the temperature is given by $\rho=$ $\rho_{0}\left[1-\alpha\left(T-T_{0}\right)\right]$, where $\alpha$ denotes the thermal expansion coefficient.

To proceed with an analytical approach, the magnetic quantities inside the fluid have to be analytically approximated. Using the corresponding results from [8], the magnetic induction (dimensionless quantities are indicated by a bar) is given by

$$
\begin{aligned}
\bar{B}_{x} & =\sin (\bar{k} \bar{x})(\mathscr{B} \bar{z}), \\
\bar{B}_{z} & =1+\cos (\bar{k} \bar{x})\left(E+G \bar{z}^{2}\right),
\end{aligned}
$$

with

$$
\bar{k}=\frac{2 \pi d}{\lambda}, \quad \mathscr{B}=-\bar{k} E, \quad G=\frac{\bar{k}^{2} E}{2} .
$$

The use of the lateral invariance in the $y$-direction allows to consider the $x$ and $z$-directions of $\bar{B}$ only. The dimensionless wave number is denoted by $\bar{k}$ and 
two of the three constants in (4), (5) are determined by the condition that the divergence as well as the vorticity of $B$ is zero. Additionally, the assumption that $G \bar{z}^{2} \ll E$ holds was used. The third constant, $E$, is a fit parameter, which yields a very good agreement with the numerical results for both components of $\bar{B}$ in the $\bar{x}$ as well as in the $\bar{z}$ direction, see Fig. 4 in [8].

Expressing the magnetic field and the magnetization in (2) with the help of $(4,5)$, the dimensionless equations for the components of $\mathbf{v}=(u, 0, w)$ and the temperature $T$ are:

$$
\begin{aligned}
& \frac{\partial \bar{u}}{\partial \bar{x}}+\frac{\partial \bar{w}}{\partial \bar{z}}=0 \\
& \frac{1}{\operatorname{Pr}}\left[\frac{\partial \bar{u}}{\partial \bar{t}}+\left(\bar{u} \frac{\partial}{\partial \bar{x}}+\bar{w} \frac{\partial}{\partial \bar{z}}\right) \bar{u}\right]=-\frac{\partial \bar{p}}{\partial \bar{x}}+\Delta \bar{u} \\
& +\operatorname{Rm} \frac{\mu_{r}-1}{2 \mu_{r}^{2}} E \bar{k} \sin (\bar{k} \bar{x})\left[E \cos (\bar{k} \bar{x})\left(\bar{k}^{2} \bar{z}^{2}-2\right)-2\right], \\
& \frac{1}{\operatorname{Pr}}\left[\frac{\partial \bar{w}}{\partial \bar{t}}+\left(\bar{u} \frac{\partial}{\partial \bar{x}}+\bar{w} \frac{\partial}{\partial \bar{z}}\right) \bar{w}\right]=-\frac{\partial \bar{p}}{\partial \bar{z}}+\Delta \bar{w}+\operatorname{Ra}\left(\bar{T}-\bar{T}_{0}\right)-\frac{\rho_{0} g d^{3}}{\kappa \eta} \\
& +\mathrm{Rm} \frac{\mu_{r}-1}{2 \mu_{r}^{2}} E \bar{k}^{2} \bar{z}\left[E\left(2+\bar{k}^{2} \bar{z}^{2}\right)+2 \cos (\bar{k} \bar{x})\right], \\
& \frac{\partial \bar{T}}{\partial \bar{t}}+\left(\bar{u} \frac{\partial}{\partial \bar{x}}+\bar{w} \frac{\partial}{\partial \bar{z}}\right) \bar{T}=\Delta \bar{T} .
\end{aligned}
$$
number

The Prandtl number $\operatorname{Pr}=\eta /\left(\rho_{0} \kappa\right)$ characterizes the fluid, where the Rayleigh

$$
\mathrm{Ra}=\frac{g \rho_{0} \alpha \delta T d^{3}}{\kappa \eta}
$$

and its magnetic counterpart [7]

$$
\mathrm{Rm}=\frac{\mu_{0} K^{2} \delta T^{2} d^{2}}{\kappa \eta}
$$

relate the destabilizing effect of the magnetic force and buoyancy, respectively, to the stabilizing effects of viscous friction and heat conduction. All lengths were scaled with $d$, the velocity with $\kappa / d$, the time with $d^{2} / \kappa$, the temperature with $\delta T$, the pressure with $(\kappa \eta) / d^{2}$, and the magnetic field with $K \delta T$, where

$$
K=-(\partial M / \partial T)_{H}
$$

is the pyromagnetic coefficient.

2. Base state. Since the Prandtl number of real magnetic fluids is large compared to unity $[9,10]$, the left-hand side of Eqs. $(8,9)$ is set to zero. As the base state is a stationary one, the solutions for the different types of modulation have to be determined for the equations

$$
\begin{aligned}
0= & -\frac{\partial \bar{p}}{\partial \bar{x}}+\Delta \bar{u}+\operatorname{Rm} \frac{\mu_{r}-1}{2 \mu_{r}^{2}} E \bar{k} \sin (\bar{k} \bar{x})\left[E \cos (\bar{k} \bar{x})\left(\bar{k}^{2} \bar{z}^{2}-2\right)-2\right], \\
0= & -\frac{\partial \bar{p}}{\partial \bar{z}}+\Delta \bar{w}+\operatorname{Ra}\left(\bar{T}-\bar{T}_{0}\right)-\frac{\rho_{0} g d^{3}}{\kappa \eta} \\
& +\operatorname{Rm} \frac{\mu_{r}-1}{2 \mu_{r}^{2}} E \bar{k}^{2} \bar{z}\left[E\left(2+\bar{k}^{2} \bar{z}^{2}\right)+2 \cos (\bar{k} \bar{x})\right], \\
0= & -\left(\bar{u} \frac{\partial}{\partial \bar{x}}+\bar{w} \frac{\partial}{\partial \bar{z}}\right) \bar{T}+\Delta \bar{T},
\end{aligned}
$$




\section{A. Lange, S. Odenbach}

augmented by Eq. (7). These equations are supplemented by rigid boundary conditions for the velocity

$$
\bar{u}=\bar{w}=0 \quad \text { at } \quad \bar{z}= \pm 1 / 2,
$$

and a constant temperature at the bottom and upper boundary, respectively,

$$
\begin{array}{lll}
\bar{T}=\bar{T}_{\mathrm{t}} & \text { at } & \bar{z}=1 / 2, \\
\bar{T}=\bar{T}_{\mathrm{b}} & \text { at } & \bar{z}=-1 / 2 .
\end{array}
$$

In the following, the base state for a long- as well as for a short-wave modulation with its characteristic flow field will be presented.

2.1. Long-wave modulation. If the modulation length of the magnetic field is large compared to the thickness of the fluid layer, i.e. $\bar{k}=k d \ll 1$, the case of long-wave modulation is given. Since this type of modulation was explicitly analyzed in [8], only the major results shall be presented here shortly. For the longwave modulation, all relevant quantities $\bar{X}(\bar{x}, \bar{z})$ are expanded in a pertubational ansatz in powers of $\bar{k}$, relevant to the $\bar{z}$ dependence of $\bar{X}$. The $\bar{x}$ dependence is modeled by $\cos (\bar{k} \bar{x}), \cos (2 \bar{k} \bar{x})$ and $\sin (\bar{k} \bar{x}), \sin (2 \bar{k} \bar{x})$, respectively. Inserting such an ansatz and solving the resulting equations in consecutive order of $\bar{k}$, the base state is finally given by $[8]$

$$
\begin{gathered}
\bar{u}_{\mathrm{b}}(\bar{x}, \bar{z})=-\left[\sin (\bar{k} \bar{x})+\frac{E}{2} \sin (2 \bar{k} \bar{x})\right] \operatorname{Rm} E\left(\mu_{r}-1\right) \frac{\left(4 \bar{z}^{2}-1\right)\left(20 \bar{z}^{2}-1\right) \bar{k}^{3}}{1920 \mu_{r}^{2}}, \\
\bar{w}_{\mathrm{b}}(\bar{x}, \bar{z})=[\cos (\bar{k} \bar{x})+E \cos (2 \bar{k} \bar{x})] \operatorname{Rm} E\left(\mu_{r}-1\right) \frac{\bar{z}\left(4 \bar{z}^{2}-1\right)^{2} \bar{k}^{4}}{1920 \mu_{r}^{2}} \\
\bar{T}_{\mathrm{b}}(\bar{x}, \bar{z})=\bar{T}_{0}-\bar{z}-[\cos (\bar{k} \bar{x})+E \cos (2 \bar{k} \bar{x})] \\
\times \operatorname{Rm} E \frac{\left(\mu_{r}-1\right) \bar{z}\left(4 \bar{z}^{2}-1\right)\left[16 \bar{z}^{2}\left(5 \bar{z}^{2}-4\right)+19\right] \bar{k}^{4}}{1612800 \mu_{r}^{2}} .
\end{gathered}
$$

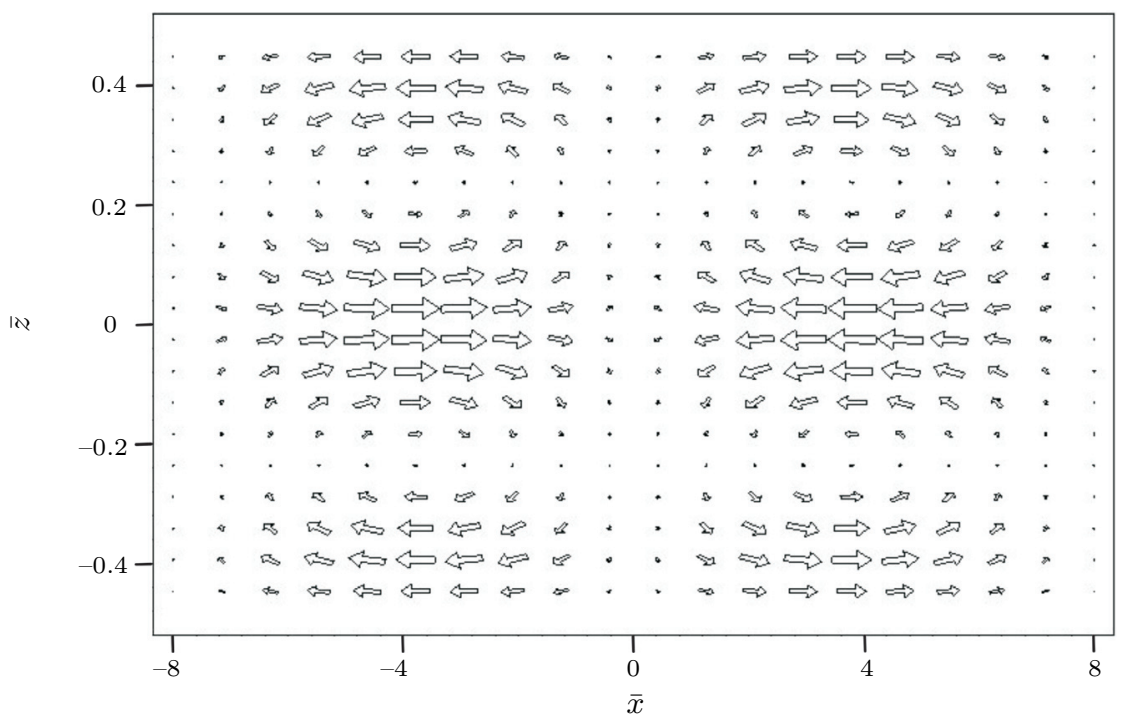

Fig. 2. Plot of the flow field for $E=0.091, \bar{k}=0.4$, and a scaled temperature difference of $\delta \bar{T}=1$ for APG $513 \mathrm{~A}\left(\rho_{0}=1.356 \times 10^{3} \mathrm{~kg} / \mathrm{m}^{3}, \alpha=4.5 \times 10^{-4} \mathrm{~K}^{-1}\right.$, $\left.\eta=150 \times 10^{-3} \mathrm{~kg} /(\mathrm{ms}), K=35 \mathrm{~A} /(\mathrm{m} \mathrm{K}), \kappa=5 \times 10^{-8} \mathrm{~m}^{2} / \mathrm{s}\right)$. With $T_{\mathrm{b}}=30{ }^{\circ} \mathrm{C}$ and $T_{\mathrm{t}}=20^{\circ} \mathrm{C}$, the Rayleigh number and its magnetic counterpart are Ra $\simeq 63.8$ and $\mathrm{Rm} \simeq 82.1$, respectively. The graph is taken from [8]. 
For a vanishing magnetic Rayleigh number, the motionless base state with a linear temperature profile inside the layer is recovered. But the qualitatively different feature compared to the classical Rayleigh-Bénard problem is the nonzero flow field (18), (19) for any nonzero magnetic Rayleigh number, see Fig. 2. For a symmetrical arrangement of bars not only a nonzero flow field exists, it has even the non-simple structure of a double vortex reflecting the symmetrical modulation of the magnetic field.

The shape of a single vortex is apparently stretched in the $x$-direction due to the modulation with a long wavelength in order to fulfill $k d \ll 1$. Thus the wavelength of the sinusoidal variation of the magnetic field strongly influences the $x$ extension of the vortex, which is much larger than the $z$ extension (see the different scales in Fig. 2). In contrast to the presented convection rolls so far, it is known from the classical Rayleigh-Bénard setup that their horizontal and vertical dimension is equal [1]. That leads to the case of short-wave modulation, which is studied in the following section.

2.2. Short-wave modulation. In the case of short-wave modulation, where $k d \sim O(1)$ or larger holds, a pertubative ansatz is no longer possible. Therefore, a much more general ansatz has to be made, which leads to a direct analytical solution of the problem. One known feature of the ansatz has to be that it recovers for $R m=0$ the quiescent base state with a linear temperature profile of the classical Rayleigh-Bénard setup. An ansatz fulfilling this constraint is given by

$$
\begin{aligned}
\bar{u} & =-\operatorname{Rm}\left[f_{1}(\bar{z}) \sin (\bar{k} \bar{x})+f_{2}(\bar{z}) \frac{E}{2} \sin (2 \bar{k} \bar{x})\right] \bar{k}^{n} \\
\bar{w} & =\operatorname{Rm}\left[g_{1}(\bar{z}) \cos (\bar{k} \bar{x})+g_{2}(\bar{z}) E \cos (2 \bar{k} \bar{x})\right] \bar{k}^{n+1} \\
\bar{T} & =\bar{T}_{0}-z+\operatorname{Rm}\left[h_{1}(\bar{z}) \cos (\bar{k} \bar{x})+h_{2}(\bar{z}) E \cos (2 \bar{k} \bar{x})\right] \\
\bar{p} & =j_{0}(z)+\operatorname{Rm}\left[j_{1}(\bar{z}) \cos (\bar{k} \bar{x})+j_{2}(\bar{z}) E \cos (2 \bar{k} \bar{x})\right]
\end{aligned}
$$

with nine unknown functions $f_{1}, f_{2}, g_{1}, g_{2}, h_{1}, h_{2}, j_{0}, j_{1}$, and $j_{2}$. This general ansatz shows that for $\bar{u}$ and $\bar{w}$ a whole set of solutions is possible since all $n \in$ $\mathbb{N}$ satisfy the constraint. Inserting the ansatz into Eqs. (7, 13-15) leads to the conditions

$$
\begin{aligned}
& f_{1}-\frac{\partial g_{1}}{\partial \bar{z}}=0 \\
& f_{2}-\frac{\partial g_{2}}{\partial \bar{z}}=0 \\
& g_{1} k^{n+1}-h_{1} k^{2}+\frac{\partial^{2} h_{1}}{\partial \bar{z}^{2}}=0 \text {, } \\
& g_{2} k^{n+1}-4 h_{2} k^{2}+\frac{\partial^{2} h_{2}}{\partial \bar{z}^{2}}=0 \text {, } \\
& -\frac{\partial j_{1}}{\partial \bar{z}}-k^{n+3} g_{1}+k^{n+1} \frac{\partial^{2} g_{1}}{\partial \bar{z}^{2}}+\frac{\left(\mu_{r}-1\right) k^{2} E \bar{z}}{\mu_{r}^{2}}+R a h_{1}=0, \\
& -\frac{\partial j_{2}}{\partial \bar{z}}-4 k^{n+3} E g_{2}+k^{n+1} \frac{\partial^{2} g_{2}}{\partial \bar{z}^{2}}+R a E h_{2}=0 \text {, } \\
& j_{1} k+f_{1} k^{n+2}-k^{n} \frac{\partial^{2} f_{1}}{\partial \bar{z}^{2}}-\frac{\left(\mu_{r}-1\right) k E}{\mu_{r}^{2}}=0, \\
& 2 j_{2} k+2 E f_{2} k^{n+2}-\frac{E}{2} k^{n} \frac{\partial^{2} f_{2}}{\partial \bar{z}^{2}}+\frac{\left(\mu_{r}-1\right) k E^{2}\left(k^{2} \bar{z}^{2}-2\right)}{4 \mu_{r}^{2}}=0 .
\end{aligned}
$$

Thereby it is assumed that in the equation of heat conduction all terms, which are quadratic in any combination of trigonometric functions, are small compared 


\section{A. Lange, S. Odenbach}

to the terms, which are linear in them. By combining these eight conditions in a cunning way, one ends with a differential equation of the 6 th order for $g_{1}$ and $g_{2}$, respectively. Since the general solution is quite lengthy to be presented here, only its principal structure is given

$$
\begin{aligned}
g_{1}= & -\frac{E k^{3}\left(\mu_{r}-1\right) \bar{z}}{k^{n} \mu_{r}^{2}\left(R a-k^{4}\right)} \\
& +C_{1} \mathrm{e}^{\alpha \bar{z}}+C_{2} \mathrm{e}^{-\alpha \bar{z}}+C_{3} \mathrm{e}^{\beta \bar{z}}+C_{4} \mathrm{e}^{-\beta \bar{z}}+C_{5} \mathrm{e}^{\gamma \bar{z}}+C_{6} \mathrm{e}^{-\gamma \bar{z}}, \\
g_{2}= & -\frac{E k^{3}\left(\mu_{r}-1\right) \bar{z}}{k^{n} \mu_{r}^{2}\left(R a-16 k^{4}\right)} \\
& +C_{7} \mathrm{e}^{\epsilon \bar{z}}+C_{8} \mathrm{e}^{-\epsilon \bar{z}}+C_{9} \mathrm{e}^{\zeta \bar{z}}+C_{10} \mathrm{e}^{-\zeta \bar{z}}+C_{11} \mathrm{e}^{\iota \bar{z}}+C_{12} \mathrm{e}^{-\iota \bar{z}},
\end{aligned}
$$

where the six different exponents $\alpha$ to $\iota$ are complicated terms by themselves. The six unknown constants in the general solution of $g_{1}$ and $g_{2}$ can be determined by

$$
g_{1,2}(1 / 2)=g_{1,2}(-1 / 2)=0, \quad \partial g_{1,2} /\left.\partial \bar{z}\right|_{1 / 2}=\partial g_{1,2} /\left.\partial \bar{z}\right|_{-1 / 2}=0,
$$

and

$$
h_{1,2}(1 / 2)=h_{1,2}(-1 / 2)=0,
$$

where the latter condition can also be expressed by $g_{1}$ and $g_{2}$.

With the knowledge of $g_{1}$ and $g_{2}$, all other remaining functions of the general ansatz (21)-(24) can be determined. In this way, for the first time an analytical solution for the base state in a spatially modulated problem beyond the region $\bar{k}=k d \ll 1$ is formulated. Since the solution enforces no restriction on $\bar{k}$, the above solution is valid in a much wider range than solutions based on perturbational approaches as given in $[8,11]$. This extended range of validity is the second main achievement beside the first one, that it is an analytical solution.

For the same set of parameters as in Fig. 2, the flow field for a short-wave modulation is calculated at the base of the functions $f_{1}$ to $g_{2}$. As Fig. 3 shows for $\bar{k}=2 \pi$, the characteristic feature of a double vortex structure remains. But the horizontal dimension of the convection roll is now of the same size as its vertical one, as one would expect from the basic convection pattern in the Rayleigh-Bénard system. Thus the case of short-wave modulation emphasizes once more that the structure of the flow patterns follows directly the width of the modulation. If one applies a long-wave modulation, the pattern is stretched in the $x$-direction resulting in rather elongated rolls. Contrary, for a short-wave modulation, the pattern is given by more circular rolls, compare Fig. 2 and Fig. 3 and note the different scales at the $x$-axes. Based on experimental observations [1], the system prefers convection patterns with equal horizonal and vertical size. Therefore, we assume that the appearance of a flow pattern according to Fig. 3 in an experiment is more likely than a flow pattern according to Fig. 2.

Studying a single convection roll for the short-wave modulation in more detail, it is apparent that the strength of the flow field in the vertical direction is of the same order as in the horizontal direction. This visual observation from the arrows in Fig. 3 is supported by the numerical data. The overall maximum of $\bar{u}$ and $\bar{w}$ is nearly the same: $\max _{\bar{x}, \bar{z}}\{\bar{u}\} \simeq 5.28 \cdot 10^{-2}$ and $\max _{\bar{x}, \bar{z}}\{\bar{w}\} \simeq 5.32 \cdot 10^{-2}$, respectively, resulting in a circular shaped roll.

The visual impression of a convection roll for the long-wave modulation, which is dominated by the horizontal flow components (see arrows in Fig. 2), is supported by the numerical data, too. The overall maximum of $\bar{u}$ is about one order of magnitude larger than the overall maximum of $\bar{w}: \max _{\bar{x}, \bar{z}}\{\bar{u}\} \simeq 5.55 \cdot 10^{-4}$ and $\max _{\bar{x}, \bar{z}}\{\bar{w}\} \simeq 3.45 \cdot 10^{-5}$, respectively, resulting in a horizontal stretched roll. 


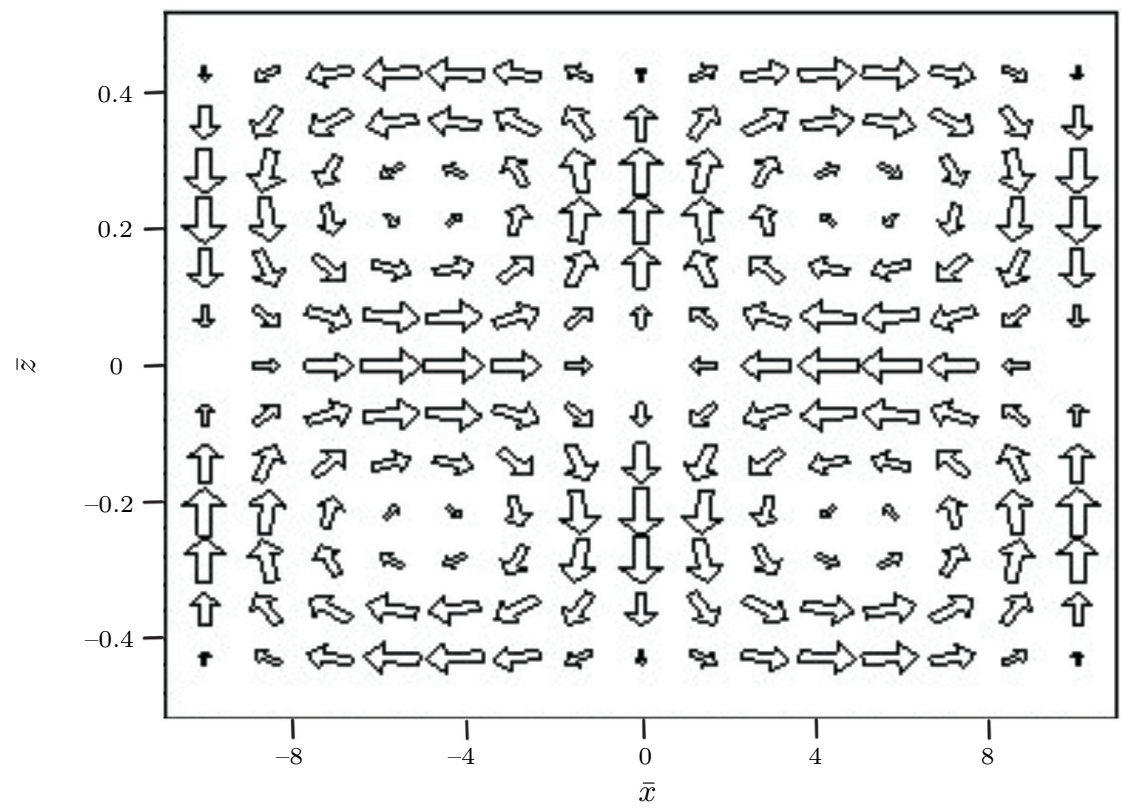

Fig. 3. Plot of the flow field for a short-wave modulation with $\bar{k}=2 \pi$. Note the same horizontal and vertical dimension of the convection roll in the double vortex structure. The remaining parameters are those from Fig. 2.

To reach the base in experiments requires two steps, in principle. In a first step, a temperature difference has to be applied across the layer that only heat conduction is present. In a second step, the magnetic field is turned on and the convective state will form. When the layer is heated from below and cooled from above, i. e. $\delta T$ is positive as considered in the governing equations, two consequences are apparent. First, the temperature difference cannot be too large even for rather viscous magnetic fluids in order to ensure the presence of heat conduction in the first step of the experimental procedure. Second, an estimation shows that the resulting temperature oscillations along the horizontal direction will be in the range of millikelvin. Reversing the temperature difference, i.e. cooling from below and heating from above, allows a greater difference in the applied temperatures, which results in larger oscillations of $T$ along the horizontal direction. But the temperature difference is limited from above by the condition for the solution $(33,34)$ that all terms, which are quadratic in the trigonometric functions, have to be small compared to the linear ones. Therefore, to find a suitable set of experimental parameters for an implementation of thermomagnetic convection caused by the short-wave modulations is a delicate task.

3. Summary. An analysis of thermomagnetic convection in a horizontal layer of a magnetic fluid subjected to a spatially and symmetrical modulated magnetic field is presented. For any nonzero magnetic field, the base state is a convective one in contrast to the classical Rayleigh-Bénard system, which is purely thermally driven. The nonzero flow field of the base state is formed by a double vortex, which reflects the symmetrical modulation from above and beneath the layer of the magnetic fluid. Depending on the type of modulation, the convection rolls forming the double vortex appear in different shapes. For the long-wave modulation, the rolls are stretched in the horizontal direction, whereas for the short-wave modulation circular rolls appear. The stability of this type of rolls, 


\section{A. Lange, S. Odenbach}

expected to be present in real experiments, against small disturbances has to be tested by a linear stability analysis. To determine the lowest threshold of stability in dependence on the parameter $n$ in equations (21) and (22) will select the right solution out of the whole set of solutions which are possible. That analysis and further details are referred to forthcoming publication.

Acknowledgements. The authors wish to thank Harald Engler for providing the material data of APG 513A.

\section{REFERENCES}

[1] M. C. Cross and P. C. Hohenberg. Pattern formation outside of equilibrium. Rev. Mod. Phys., vol. 65 (1993), no. 3, pp. 851-1112.

[2] S. Chandrasekhar. Hydrodynamics and hydrodynamic stability (Dover Publ., New York, 1981).

[3] T. Völker, E. Blums, and S. Odenbach. Heat and mass transfer phenomena in magnetic fluids. GAMM-Mitt., vol. 30 (2007), no. 1, pp. 185-194.

[4] L. Schwab, U. Hildebrandt, and K. Stierstadt. Magnetic Bénard convection. J. Magn. Magn. Mat., vol. 39 (1983), pp. 113-114.

[5] L. Schwab and K. Stierstadt. Field-induced wavevector-selection by magnetic Bénard-convection. J. Magn. Magn. Mat., vol. 65 (1987), pp. 315-316.

[6] L. Schwab. Thermal convection in ferrofluids under a free surface. J. Magn. Magn. Mat., vol. 85 (1990), pp. 199-202.

[7] H. Engler and S. Odenbach. Parametric modulation of thermomagnetic convection in magnetic fluids. J. Phys.: Condens. Matter, vol. 20 (2008), no. 20 , p. 204135 .

[8] A. Lange and S. Odenbach. Patterns of thermomagnetic convection in magnetic fluids subjected to spatially modulated magnetic fields. Phys. Rev. $E$, vol. 83 (2011), no. 6, p. 066305.

[9] S. A. Suslov. Thermomagnetic convection in a vertical layer of ferromagnetic fluid. Phys. Fluids, vol. 20 (2008), no. 8, p. 084101.

[10] P. N. Kaloni and J. X. Lou. Convective instability of magnetic fluids. Phys. Rev. E, vol. 70 (2004), no. 2, p. 026313.

[11] R. Schmitz And W. Zimmermann. Spatially periodic modulated RayleighBénard convection. Phys. Rev. E, vol. 53 (1996), no. 6, pp. 5993-6011. 